Relación entre agroindustrias y empresas agrocomerciales con la explotación primaria agrícola en el área aledaña Marina Bustamante, Diego Fabián Zalazar, Ricardo Oscar Agüero

\title{
RELACIÓN ENTRE AGROINDUSTRIAS Y EMPRESAS AGROCOMERCIALES CON LA EXPLOTACIÓN PRIMARIA AGRÍCOLA EN EL ÁREA ALEDAÑA A LA CIUDAD DE RÍO CUARTO, REPÚBLICA ARGENTINA. MODIFICACIONES SOCIO-TERRITORIALES
}

\author{
Relation between agriculture industries and agriculture business companies with the \\ agricultural primary exploitation in the bordering area of Río Cuarto city, República \\ Argentina socioterritorial modifications
}

\begin{abstract}
Marina Bustamante
Diego Fabián Zalazar

Ricardo Oscar Agüero

Universidad Nacional de Río Cuarto, Facultad de Ciencias Humanas, Departamento de Geografía. Ruta Nac. 36, Km. 601, Río Cuarto, Córdoba, Argentina. CP X5804BYA E-mail: mbustamante@hum.unrc.edu.ar,dzalazar@hum.unrc.edu.ar, roaguero2002@yahoo.com.ar
\end{abstract}

Artigo recebido para publicação em 25/03/08 e aceito para publicação em 20/05/08

RESUMEN: En los últimos años se ha asistido a una profunda transformación en la estructura económica de nuestro país, que ha traído aparejado un fuerte desdibujamiento de los roles desempeñados por los agentes laborales tradicionales, llevando a agudizar las desigualdades existentes entre los actores implicados. El presente trabajo -circunscripto al área aledaña a la ciudad de Río Cuarto, en la Región Pampeana argentina- constituye una aproximación a la injerencia que tienen las agroindustrias y empresas agrocomerciales sobre la actividad primaria agrícola mediante la explotación de tierras en propiedad, arrendadas, en sociedad, etc, conformando para ello asociaciones de diversa índole entre los diferentes actores del mundo rural a través de un complejo entramado de relaciones socio-económicas.

Nuestro principal propósito es detectar el peso económico de las agroindustrias y empresas agrocomerciales sobre la estructura agraria, enfatizando en sus formas operativas de trabajo con respecto a la explotación primaria agrícola y en los efectos socioeconómicos que desencadena, especialmente entre los pequeños productores. A su vez, se procura desentrañar el rol y las estrategias de adaptación desarrolladas por estos últimos. Por otra parte, se analizan las progresivas transformaciones espaciales producidas como consecuencia de estos procesos.

Las transformaciones manifestadas han llevado a intensificar los procesos de integración, evidenciando un significativo cambio de roles de los actores tradicionales y la incorporación de nuevos agentes con tendencia hegemónica sobre los anteriores. Este fenómeno va acompañado de inusitados incrementos en la producción e importantes desigualdades sociales y desarticulaciones territoriales.

Palabras claves: Río Cuarto, agroindustrias, empresas agrocomerciales, estructura agraria, modificaciones socio-territoriales.

SUMMARY: In the last years we have attended a deep transformation in the economic structure of our country, that has brought about a strong blurring of the roles carried out by the traditional labor agents, whose

Sociedade \& Natureza, Uberlândia, 20 (2): 121-133, DEZ. 2008 
consequences have worsen the prevailing inequalities between the implied actors.

The present work-circumscribed to the area bordering the city of Río Cuarto, in the Argentine Pampean Region-constitutes an approach to the interference which agriculture industries and agriculture business companies have on the primary agricultural activity by means of the exploitation of lands owned, rented, in society, etc. To achieve this purpose associations of diverse nature among the different actors from the rural world have been set up through a complex framework of socioeconomic relations.

Our main aim is to detect the economic power of agriculture industries and agriculture business companies on the farm structure, emphasizing in its operative forms of work in relation to the agricultural primary exploitation and in the socioeconomic effects that it triggers, specially among the small producers. As well, we try to unravel the roll and the strategies of adaptation developed for these producers. On the other hand, the progressive space transformations produced as a result of these processes are analyzed. The stated transformations have taken to intensify the integration processes, demonstrating a significant change of roles of the traditional actors and the incorporation of new agents with hegemonic tendency on the previous ones. This phenomenon is accompanied of unusual increases in the production and important social inequalities and territorial disarticulations.

Key words: Río Cuarto, agriculture industries, agriculture business companies, farm structure, socioterritorial modifications.

\section{INTRODUCCIÓN}

El tema que aquí se expone, consiste en una aproximación indagatoria sobre la injerencia y formas de trabajo de las agroindustrias y empresas agrocomerciales, en general sobre la actividad primaria agrícola, mediante la explotación directa de tierras en propiedad y arrendadas, bajo acuerdo de partes y en relación societal, conformando para ello vinculaciones de diversa índole entre los diferentes actores del mundo rural, a través de un complejo entramado de relaciones socio-económicas.

El área de estudio, que tiene como pivote a la ciudad de Río Cuarto, corresponde a un sector representativo de la llanura pampeana y comprende específicamente el sur de la provincia de Córdoba, República Argentina (Mapa $\mathrm{N}^{\circ}$ 1). Corresponde a un territorio sumamente significativo que, sin pertenecer estrictamente al área nuclear -o sea de mayor productividad- de la Región Pampeana, reúne condiciones óptimas especialmente para los cultivos granarios de verano y para las actividades agropecuarias de clima templado en general.
Es pertinente acotar que, por similitudes geográficas, los fenómenos socio-económicosespaciales que se manifiestan en nuestra área de estudio generalmente se reproducen, en mayor o menor medida, en toda la llanura pampeana.

A continuación, nos parece oportuno, antes de entrar en las especificidades de nuestra temática, contextualizar la serie de avatares por la que viene atravesando el campo argentino en los últimos tiempos.

En este sentido, vemos que especialmente desde la década del '90 se viene gestando una profunda transformación en la estructura económica agropecuaria de nuestro país en consonancia con el resto de la economía nacional, fenómeno que se manifiesta también en mayor o en menor escala a nivel mundial.

Este proceso de cambios, que en sí viene de décadas anteriores, en los noventa se incrementa con particular virulencia, mediante la intromisión a rajatabla de políticas neoliberales, que van a implicar la desregulación de los controles económicos por parte del Estado y su reemplazo por las leyes directrices

Sociedade \& Natureza, Uberlândia, 20 (2): 121-133, DEZ. 2008 
del mercado; va a producir en el ámbito rural, entre otras consecuencias, un fuerte desdibujamiento de los roles desempeñados por los actores laborales tradicionales y la incorporación de nuevos agentes con tendencia hegemónica sobre los anteriores y va a repercutir con especial énfasis en la estructura agraria, desencadenando fuertes modificaciones socioterritoriales.

Estas transformaciones estructurales generales que venía sufriendo el país van a eclosionar mediante una severa crisis económica a fines del año 2001, produciendo un inusitado endeudamiento financiero nunca visto en periodos históricos anteriores. En este estado de indefensión económica, el primer sector laboral que va a reaccionar positivamente corresponde al ámbito rural, que a través de los años siguientes se afianza sólidamente, generando espectaculares incrementos en la producción y convirtiéndose en el motor económico que logra sanear paulatinamente las finanzas del país, pero que por otra parte está lejos de saldar los desequilibrios socio-económicos que viene arrastrando la sociedad argentina.

Pero a pesar del estado de buena salud del campo y la bocanada de oxígeno que éste vierte a toda la economía de la nación, el mismo encubre o no refleja en realidad las contradicciones y alteraciones que se vienen produciendo dentro de la estructura interna de los agentes productivos agropecuarios y de la estructura agraria en general.

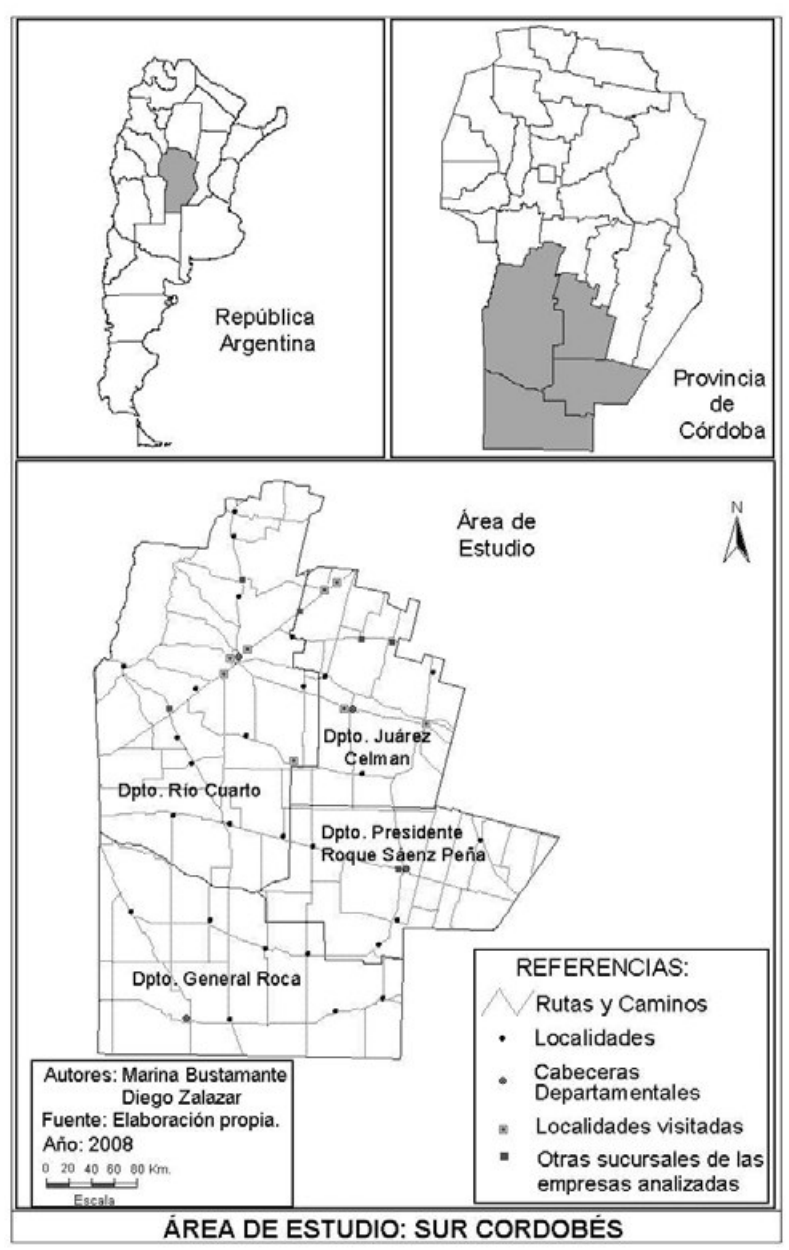

$\operatorname{Mapa} N^{\circ} 1$ 
Es por ello que nos interesa indagar sobre la intromisión -en nuestro medio geográfico- de los complejos agroindustriales en la producción agropecuaria, por entender que dichas organizaciones tienen mucho que ver en la desarticulación del sistema agropecuario precedente, que sin ser perfecto, estaba aparentemente más equilibrado desde el punto de vista socio-económico que el actual. Por otra parte, constatamos que la incidencia de las agroindustrias en la producción agraria no es un fenómeno nuevo, sino que se viene manifestando desde hace varias décadas en nuestro ámbito nacional.

A su vez, es digno de apreciar que son varios los autores y numerosos los trabajos que han analizado este fenómeno (TEUBAL Y PASTORE, 1995; GUTMAN Y GATTO, 1990; TEUBAL Y RODRÍGUEZ, 2003), sin que por ello se termine de estudiar esta problemática. Motivo por el cual nos hemos animado a investigar, por un lado, el accionar de estos complejos - a título de estudio de caso- en nuestra región, y por el otro, por considerar que es en cierto modo una "novedad" interesante de profundizar que: 1) el nexo en la "integración asociativa" se establece actualmente entre empresa-productor individual (variante vertical), y no a través de pools de siembra (variante horizontal) -como se daba especialmente en la década anterior-donde intervienen varios agentes, muchos de ellos ajenos al métier agropecuario y donde se prioriza sobre todo el volumen del aporte financiero, y 2) que dicha integración entre los actores básicamente se manifiesta principalmente a partir de la crisis económica 2001-2002.

En síntesis, consideramos que la injerencia utilizando diferentes estrategias- de los complejos agroindustriales y empresas conexas en la producción "primaria" contribuye a incrementar las asimetrías socio-territoriales (de claras consecuencias negativas), que cada vez en forma más nítida se ponen de manifiesto en el campo argentino.

\section{OBJETIVOS Y METODOLOGÍA}

Nuestro principal propósito es detectar el accionar y el peso económico de las agroindustrias y empresas de comercialización de granos sobre la estructura agraria, y desentrañar a su vez el modo en que las mismas extienden su dominio hacia la actividad agrícola mediante diferentes modalidades de integración vertical.

Asimismo, se procura captar los diversos roles y estrategias de adaptación desarrollados por los productores vinculados con los complejos agroalimentarios.

Por último, se analizan las progresivas modificaciones socio-territoriales resultantes.

Para la consecución de estos objetivos, en esta oportunidad, nos basamos en el análisis de material bibliográfico pertinente, a fin de diseñar un marco teórico conceptual y principalmente en el trabajo de campo, con el propósito de obtener información de primera mano, razón por la cual visitamos diversas localidades de la zona bajo estudio. Para ello, se realizaron entrevistas sistematizadas a personas ligadas a las actividades agropecuarias mediante la confección de diversas guías de entrevistas, que se adecuaron según las características específicas de los interlocutores. Asimismo, para el relevamiento de campo se elaboraron guías estandarizadas, a fin de volcar en éstas de manera ordenada la información oral recogida, datos provenientes de material cartográfico e impresiones de lo observado directamente, con el objetivo -a nivel aproximativo en esta instancia de la investigación- de cualificar variables centrales que ayuden a construir el marco estructural del trabajo, de modo de arribar a la comprensión de la situación actual $\mathrm{y}$ detección de posibles tendencias a futuro.

A continuación pasamos a describir las diferentes formas y características de integración vertical "hacia atrás" (o sea, sobre la producción agropecuaria), que emplean las agroindustrias y empresas agrocomerciales relacionadas con la actividad granaria localizadas en nuestra región, de acuerdo con lo observado en nuestras investigaciones.

Sociedade \& Natureza, Uberlândia, 20 (2): 121-133, DEZ. 2008 
Formas de integración vertical hacia la actividad primaria de las agroindustrias y empresas agrocomerciales que se desempeñan en nuestro medio

\section{Integración vertical directa (o propiamente dicha)}

Corresponde cuando la empresa controla en forma directa la producción primaria, o sea, explota los campos por cuenta propia bajo su responsabilidad y riesgo.

Para llevar a cabo este tipo de integración vertical "hacia atrás", dispone de tierras en propiedad y/o de tierras arrendadas. Los agentes laborales que trabajan este campo pueden ser:

- empresas de explotación propia, que dependen del Departamento de Campo de la firma;

- contratistas de servicios;

- productores, en el rol de contratistas de servicios.

Tanto en el segundo caso como en el tercero, se les paga específicamente por el servicio que prestan, o sea, por el trabajo realizado.

Corresponde aclarar, a título general, que todas las empresas que se integran a la producción agropecuaria cuentan con un Departamento de Campo encargado de dirigir la actividad productiva, que en algunos casos constituyen firmas independientes ligadas obviamente a las empresas, pero legalmente autónomas, por razones operativas y económicas. En general, asesoran y dirigen las explotaciones, pero no cuentan con maquinaria propia; no obstante, en algunos pocos casos, sí cuentan con equipo de laboreo propio.

En concreto, tanto en las tierras en propiedad como en las arrendadas a terceros, la dirección, administración y control está a cargo de la empresa $\mathrm{y}$, por consiguiente, no comparte los beneficios ni los riesgos con otros agentes productivos.
Esta modalidad de integración permite aplicar técnicas evolucionadas de laboreo y contar asimismo con calidad y previsibilidad en los volúmenes productivos requeridos.

\section{Integración vertical indirecta (o de acuerdo de partes)}

En este tipo de investigación, la empresa no dirige las explotaciones sino que las mismas pertenecen a los productores, que son quienes las dirigen y trabajan. Éstos se desempeñan laboralmente de manera independiente, o sea, trabajan por cuenta propia. Lo que sí se establece entre las partes es una suerte de acuerdo por medio del cual la empresa en general adelanta los insumos (semillas y agroquímicos) y el productor, que pone la tierra y el trabajo, devuelve el préstamo en granos. Este tipo de operatoria se suele denominar "contrato de entrega de granos". La empresa no participa de los riesgos sino que éstos corren por cuenta del productor que se desempeña en forma autónoma. Lo que se establece es un "canje" que en algunos casos puede consistir en una especie de ayuda económica al productor, comprometiéndose éste a hacer entrega del producto cosechado.

El adelanto de insumos por parte de la empresa va acompañado, en general, por exigencias al productor en lo que atañe a la calidad y fecha estimativa de entrega del producto.

En nuestra área de estudio, normalmente estos acuerdos son de palabra, sin mediar contratos por escrito. Ésta es una característica que posiblemente marca la diferencia de la aparente integración similar (o equivalente) a la que Teubal denomina "integración vertical contractual o agricultura de contrato", en su clasificación sobre formas de integración vertical en algunos complejos agroindustriales (TEUBAL Y PASTORE, 1995).

En síntesis, entendemos que la incidencia de las empresas en este tipo de integración es indirecta, el responsable y el que arriesga es indudablemente el productor; no obstante, gran parte de la orientación y 
resultados productivos - en forma mediatizada- son canalizados a través de las empresas, con las ventajas para las mismas de poder contar en una fecha aproximada con un stock de mercadería determinada y calidad prevista. A su vez, la ventaja para el productor-que puede ser relativa y de consecuencias imprevisibles si el grado de dependencia económica es agudo- consiste en poder contar con una especie de crédito para iniciar la campaña agrícola, asesoramiento técnico en muchos casos y eventualmente precios previsibles y garantía de la venta de la producción.

\section{Integración vertical asociativa}

En esta relación, la empresa por una parte y un agente productivo por otra conforman una "sociedad" de explotación de la tierra. Las dos partes intervienen en el control de la explotación, comparten los riesgos y reparten la producción proporcionalmente, de acuerdo con lo que aporta cada uno en el vínculo societal.

El productor generalmente aporta la tierra y las labores, y la empresa los insumos, el asesoramiento técnico y eventualmente el transporte. El reparto de los beneficios se hace proporcionalmente, 0 según la contribución realizada a la producción. Se trabaja normalmente con el "contrato de aparcería". Cabe aclarar que lo que se reparte son los frutos y no las ganancias, o sea, al momento de repartir los beneficios, lo que se reparte es la producción.

En este tipo de integración, las empresas intervinientes trabajan en la mayoría de los casos con los productores individuales y no con otras empresas. Solamente en unas pocas circunstancias o en situaciones coyunturales, por ejemplo de tener que cumplir con un pedido de compra de mercadería de grandes proporciones, se lleva a cabo una relación societal de tipo interempresarial.

Esta nueva estrategia de las agroindustrias que parece reemplazar a los clásicos pools de siembra- consistente en entablar sociedades con un sinnúmero de productores, parece ser la modalidad predominante y más novedosa de integración vertical hacia atrás que se da en nuestro medio geográfico (sur de la provincia de Córdoba), donde todos salen ganando, pero las empresas en mayor proporción, utilizando diversas variantes de contratos según las posibilidades de los productores.

Corresponde especificar que por lo general los aportes que realizan las empresas en esta vinculación societal son mayores que los que disponen los productores, por lo tanto obviamente son mayores los beneficios, aparte de otras ventajas que obtienen a partir de la sumatoria de asociaciones que establecen.

Por otra parte, los productores deben reunir ciertas condiciones de solvencia a juicio de las empresas, como ser preferentemente propietarios de explotaciones de regular a gran tamaño; disponer de maquinaria o parte de la misma adecuada a la siembra directa, y manejar en general las modernas técnicas agropecuarias.

Existe una gran variedad de formas de asociación que se adecuan a la situación económica de cada propietario, pero aparte de la clásica vinculación arriba descripta entre el productor y la empresa, donde el primero aporta la tierra y las labores, en muchos casos es la misma empresa la que contribuye con tierras, mediante el arrendamiento de campos -o parte de ellos-, ya sea a los mismos productores asociados -en esta circunstancia éstos van a percibir una cantidad menor de beneficios- o a terceros. También puede darse el caso de que la empresa y el productor, en forma conjunta, arrienden tierras a terceros.

En estas situaciones donde media el alquiler de tierras, los contratos de arrendamientos que se establecen son todos por escrito; en cambio, los aportes que realizan en la vinculación societal cada una de las partes se acuerdan de palabra.

Por último, cabe aclarar también que la diferencia de este tipo de integración societal descripta con respecto a la que denomina casi del mismo modo 
Teubal ("Integración vertical asociativa y cooperativizada") en su clasificación sobre la integración de los complejos agroindustriales ya mencionada, es que él se refiere básicamente a un tipo de asociación entre varias unidades de producción del sector primario que "se articulan horizontalmente para participar en otra etapa e integrar verticalmente sus respectivas producciones $\mathrm{y} / \mathrm{o}$ procesos productivos" (TEUBAL Y PASTORE, 1995, p.120); en cambio, en nuestro caso, la vinculación es entre dos agentes productivos, el productor y la agroindustria que integra a éste. En suma, en general es éste el tipo de vinculación societal de por sí asimétrica el que se manifiesta en nuestro medio y no aquel al que hace referencia Teubal, lamentablemente.

El nivel de asociaciones de productores y de vínculos cooperativizados en defensa de la producción granaria está muy poco desarrollado en nuestra zona de estudio.

\section{Características generales y desempeño en el proceso de integración vertical hacia atrás de algunas de las agroindustrias y agrocomercios más representativos de nuestro medio dedicados a la industrialización y comercialización de granos}

Seguidamente, pasamos a describir, en forma sucinta, las características y desempeño direccionados hacia la actividad agrícola de algunas de las mayores empresas agroindustriales y agrocomerciales más representativas de nuestro medio, que forman parte, a su vez, del complejo cerealero y aceitero argentino.

Es pertinente aclarar que las empresas a las cuales hemos analizado, en general están integradas por un conjunto de firmas autónomas, pero subsidiarias de una empresa madre y que en conjunto desempeñan dentro del sector agropecuario múltiples funciones económicas, en el rubro industrial, primario, comercial, de servicios y financiero, constituyendo en última instancia verdaderos "grupos empresariales".

No obstante la diversidad de actividades económicas que detentan las empresas arriba mencionadas, en este acápite las diferenciamos por la actividad económica por la que se la distingue en nuestro medio, englobándolas en conjunto por similitud de desempeño productivo, tanto las correspondientes a la actividad agroindustrial como agrocomercial.

\section{Empresas agroindustriales de oleaginosas y cereales}

Con respecto a la actividad agroindustiral granaria en nuestra zona, se destaca la aceitera y molinera. Dentro de la primera sobresale una firma que está catalogada como una de las empresas más importantes del país, que si bien la empresa madre se ubica en nuestra área de estudio, sus plantas industriales y de acopio se distribuyen en numerosas provincias de la nación.

El complejo agroindustrial aceitero que se centra en nuestro medio cubre todo el circuito productivo desde la actividad industrial, pasando por la primaria hasta la comercialización de la producción aceitera y de diversos productos derivados de semillas oleaginosas y cerealeras. El proceso integrador a partir del núcleo agroindustrial, tanto hacia atrás como hacia adelante, está sumamente desarrollado y consolidado.

En lo atinente a la agroindustria molinera, la misma está bastante extendida en nuestra zona y una de las materias primas básicas que utiliza es el trigo. Una de las principales firmas está representada por un grupo agroindustrial de extensas ramificaciones que se prolonga también en diversas provincias del país. Cuenta con molinos harineros, campos propios, plantas de acopio, fábricas de productos alimenticios, empresas prestadoras de servicios agropecuarios, etc., pero en nuestro medio se destaca como agroindustria molinera. Representa a un grupo empresarial sumamente diversificado, dueño de distintas firmas que se integran entre sí, pero cada una actúa de manera autónoma.

Cabe aclarar que dentro de la cadena productiva de las agroindustrias mencionadas, la injerencia operativa en la actividad agrícola en la industria aceitera está a cargo de un Departamento 
de Campo, encargado de la parte técnica, pero no cuenta con maquinaria propia; por lo tanto, las labores están tercerizadas. En cambio, en el caso de la industria molinera, la dirección del manejo de la empresa está a cargo de una firma subsidiaria de la empresa madre, que sí cuenta con maquinaria propia y ella misma lleva a cabo en gran parte las labores agropecuarias.

Con respecto a las diferentes formas de integración vertical que aplican las mencionadas agroindustrias, tanto una como la otra practican la integración vertical directa. La agroindustria aceitera, para ello, dispone tanto de tierras en propiedad como arrendadas, que ocupan extensiones más que importantes. Las mismas son trabajadas por contratistas de servicios o productores que cumplen un rol similar al de los contratistas.

La agroindustria molinera, en cambio, trabaja casi exclusivamente tierras en propiedad, que también ocupan grandes extensiones, y las labores de las mismas se llevan a cabo mediante explotación propia a través de la empresa subsidiaria destinada a tal fin. La integración vertical indirecta la aplica solamente la agroindustria aceitera, donde media en muchos casos un contrato de entrega de granos y donde este intercambio de integración opera como un canje entre las partes.

La integración vertical asociativa actúa en los dos tipos de agroindustrias, pero en la aceitera solamente opera -a gran escala-con los productores, bajo la figura del contrato modelo de aparcería; en cambio, en la molinera, los vínculos asociativos se establecen entre empresas, o sea, a nivel de empresas de grandes dimensiones económicas.

Las tierras trabajadas por ambas agroindustrias se encuentran dispersas por todo el sur cordobés, especialmente la aceitera que se ha extendido sobremanera hacia el sur, hasta traspasar el límite establecido entre las provincias de Córdoba y La Pampa.

Los cultivos que practica la agroindustria molinera están muy diversificados y tanto los de invierno como los de verano son relevantes, pero lo que produce en mayor proporción, como es de esperar, es la soja (60\%), seguida por el maíz, el maní, el girasol, el trigo, etc.

En el caso de la agroindustria aceitera, los granos que cultiva son: soja, que ocupa un lugar destacado, girasol, maní (que oficia como materia prima tradicional de esta industria), trigo y maíz.

En ambas agroindustrias la producción granaria que las alimentan proviene de campos laborados con siembra directa y las semillas que se utilizan, en gran parte producidas por la misma empresa, son las genéticamente modificadas. Los campos con los cuales trabajan reciben asesoramiento tecnológico y controles de calidad.

La política de la agroindustria molinera en relación con la producción primaria se basa, por un lado, en no arrendar campos a terceros, o sea, la idea es trabajar con campos propios de la firma; por otro, diversificar en todo lo posible la producción para disminuir los riesgos.

Por otra parte, la política de la agroindustria aceitera propicia los siguientes criterios: que cada planta de acopio de su propiedad se maneje en su zona con total autonomía de decisión, a través del gerente de planta; no comprar tierras pero sí arrendar más. No obstante, las políticas a largo plazo no apuntan a salir a buscar tierras para arrendar, sino que los productores se acerquen a la agroindustria con el objetivo de asociarse.

\section{Empresas agrocomerciales de acopio $y$ comercialización de granos}

En nuestra zona de estudio son varias las organizaciones que se dedican al acopio y a su vez tienen injerencia en la producción primaria, pero en este trabajo tomamos como ejemplo una empresa acopiadora de importancia zonal que cuenta con numerosas sucursales en la región, y que además funciona como distribuidora de insumos y de

Sociedade \& Natureza, Uberlândia, 20 (2): 121-133, DEZ. 2008 
combustible. Asimismo, incluimos en el análisis a una cooperativa muy consolidada en nuestro medio, que cuenta también con varias sucursales, pero con un área de influencia relativamente restringida, que no cubre toda la región. Por último, también incluimos en este análisis la descripción, a modo de ejemplo, de una planta de acopio modelo, ubicada en nuestra zona, dependiente de una agroindustria aceitera líder en la que, al poder desempeñarse con total autonomía de decisión en lo que respecta a las relaciones con los productores, el gerente de planta puede entablar diferentes vinculaciones de integración con los agentes productivos.

El sector responsable de la dirección de la producción primaria en la empresa acopiadora está a cargo de una firma subsidiaria de la misma, y en el caso de la cooperativa y de la planta de acopio modelo, la responsabilidad recae en los respectivos Departamentos de Campo habilitados a tal fin. Ninguna de estas tres instancias organizativas cuenta con maquinaria propia, por lo tanto las labores también se tercerizan.

En relación con los tipos de integración vertical que se implementan, en las dos primeras organizaciones nombradas se aplica la integración vertical directa, no así en cambio en la planta de acopio modelo. En la empresa acopiadora se realiza a través del empleo de tierras en propiedad y arrendadas, y son trabajadas por contratistas de servicios; en cambio en la cooperativa se lleva a cabo solamente en tierras arrendadas (donde se siembra exclusivamente maní) y son trabajadas por contratistas de servicios. En los dos casos mencionados, las tierras laboradas bajo esta forma de integración no abarcan extensiones de grandes dimensiones.

La integración vertical indirecta no se aplica en la cooperativa, pero sí en la empresa acopiadora, donde media en muchos casos una suerte de contrato de siembra, y también en la planta de acopio modelo, cuya aplicación aquí sobre todo cumple la función de ayuda económica al productor, quien debe devolver con la producción.
Por último, la integración vertical asociativa se manifiesta en las tres organizaciones nombradas, y es la que tiene mayor difusión dentro de las diferentes formas de integración. A su vez, las tres organizaciones económicas trabajan exclusivamente con productores, donde el vínculo que se establece de hecho es por demás asimétrico. En este sentido, queda pendiente un análisis más en profundidad con respecto a la vinculación asociativa que se establece entre la cooperativa analizada y los productores con los que se asocia. Por ahora da la impresión de que la misma se desempeña más como empresa, que como asociación de productores que se unen para alcanzar ventajas y beneficios económicos equitativamente compartidos.

La localización geográfica de las empresas agrocomerciales citadas se esparcen también por todo el sector centro-sur de la provincia de Córdoba, pero especialmente próximas a las sucursales respectivas.

La empresa acopiadora, en los campos arrendados, cultiva un $70 \%$ de soja, $20 \%$ de maíz y $10 \%$ de girasol. En los campos propios se trata de hacer la siguiente rotación: $33 \%$ de soja de primera, $33 \%$ de maíz y 33\% de trigo-soja de segunda. Queda claro que en las tierras propias, no así en las ajenas, media un criterio conservacionista, con el objetivo de no agotar el suelo y mejorar su productividad.

En el caso de la cooperativa, ocupa un lugar importante el cultivo de maní, debido a que cuenta con una planta industrial seleccionadora de este grano a la que debe abastecer. También ocupa un lugar relevante el acopio de soja, seguido por otros granos como el maíz y el girasol.

En la planta acopiadora modelo, el principal cultivo corresponde a la soja, seguido por el maíz, el girasol y el maní.

En la producción granaria que alimenta a todas las empresas vinculadas al acopio se aplica la siembra directa, se utilizan semillas mejoradas y se lleva a cabo el correspondiente control de calidad. 
En relación con la política a futuro de la empresa de acopio, ésta consiste en especializarse más en la parte comercial que en la productiva, es decir, tender más a una integración hacia adelante.

Por otra parte, no piensa comprar más tierras, sino arrendar campos con contratos por períodos más prolongados, para tener así una mayor continuidad sobre las tierras. Actualmente tiene establecidos contratos por una sola campaña y la idea es aumentar el período a tres años, para el bien de todos.

En lo que respecta a la política que sustenta la cooperativa, considera que, si bien tiene que sembrar maní por cuenta propia, a fin de garantizar una cantidad fija de materia prima para abastecer a su planta seleccionadora, entiende que la tarea de sembrar no es su función, sino que esa actividad le corresponde al productor.

\section{CONCLUSIONES}

En la integración que se establece entre el agro y la industria en nuestro medio, queda claro que los beneficios y ventajas que obtienen las agroindustrias y agrocomercios granarios -donde éstos últimos están estrechamente ligados a las primeras-pasa, en primer lugar, por garantizar la cuota de materia prima que requiere el procesamiento industrial, y no dejar librado a los vaivenes del libre mercado el aprovisionamiento de los insumos necesarios y, en segundo lugar, por obtener un mejor precio de las materias primas requeridas, al evitar o disminuir la intermediación, al tener acceso directo a la producción primaria.

Con respecto a la otra cara de la vinculación, o sea la de los productores agropecuarios, conviene recordar que la asociación con las empresas en nuestra zona surge o se acrecienta fundamentalmente a partir de la crisis y devaluación de 2001-2002, cuando a un número considerable de productores, por endeudamiento y/o por falta de capital, se les dificultó seguir trabajando de manera independiente. Surgió entonces la posibilidad de establecer nuevas estrategias, tales como asociaciones de diversa índole con empresas del medio. En cierto modo, las agroindustrias -durante ese período de crisis- se ocuparon de suplir la falta de crédito por parte de diversos organismos.

Ahora bien, no todos los agricultores estuvieron o están en situación de poder cubrir las condiciones técnico-financieras que el ritmo económico digitado desde la cúspide de la cadena agroindustrial exige actualmente a la producción del campo, donde se debe en forma pareja reunir calidad y precios competitivos de las materias primas, rendimiento apreciable por unidad de superficie y fechas de entrega de la producción en tiempos estimativos, entre otras exigencias más, lo que determina obviamente que los productores más desvalidos no puedan competir.

Por otra parte, más allá de los requerimientos que actualmente solicitan las empresas a los productores que sí pueden reunir las condiciones de exigencia nombradas, entran ahora también en el juego de las relaciones asociativas productores capitalistas, de claro perfil empresarial, de gran capacidad económica, que en muchos casos no figuran como productores clásicos ni como dueños de explotaciones.

En concreto, el rol que van a desempeñar los productores de características tradicionales vinculados a las agroindustrias es básicamente el de socios menores y dependientes de las mismas. El asociarse implica, por un lado, una forma de subsistir, y por otro, poder permanecer dentro del sistema productivo, compitiendo gracias al auxilio del "hermano mayor". Por cierto, ganan todos, pero el productor en menor proporción.

Además, es pertinente destacar que dentro de las formas de integración vertical -según nuestra clasificación- que se manifiestan en nuestro medio, predomina la integración vertical asociativa que, por sus características, determina que los vínculos que se establecen entre las partes implican para el productor una fuerte dependencia general y una marcada asimetría relacional. 
De acuerdo con lo expuesto, se plantea un fenómeno dialéctico, en el sentido de que, por un lado, las agroindustrias cumplen la función de salvataje o ayuda para mantener en actividad a ciertos estratos de productores, y por otro, su injerencia en la producción primaria agudiza la asimetría y la concentración de capital en el campo, lo que a la larga repercute negativamente en el grueso de los productores agropecuarios y, por carácter transitivo, en los asalariados rurales, al imponer criterios hegemónicos y competitivos que condicionan las potencialidades económicas a futuro y coartan la independencia productiva de las explotaciones.

Todas estas contradicciones están dentro y forman parte de un contexto más amplio, que tiende a incrementar el aposentamiento y consolidación de un sistema capitalista avanzado de control del capital sobre la tierra, donde los individuos y las relaciones socio-económicas tradicionales son arrasadas, imponiendo la despersonalización y la obtención de la mayor tasa de ganancia posible a costa de forzar técnicas y naturaleza.

Volviendo a nuestra región de estudio, observamos que este fenómeno de relación asimétrica entre las partes, empresas-productores, de seguir así, puede llegar a producir en un sentido amplio una disminución numérica más pronunciada de los pequeños y medianos productores familiares, dado que las agroindustrias, al optimizar la producción del campo convirtiéndola en altamente competitiva, por una parte, y preferir en la relación societal a los productores acomodados o capitalistas, por otra, determinan que los mismos queden acorraladas entre dos fuegos. Esto lleva a su vez a un incremento de la disponibilidad de tierras en arrendamiento de parte de aquellos propietarios, que no disponen de los medios suficientes para encarar de manera independiente o asociativa la explotación de sus campos; en cambio, de este modo sí pueden obtener una buena renta. Cabe aclarar que en esta circunstancia, el agricultor deja de ser productor para convertirse en rentista.

Con respecto a las consecuencias socioterritoriales que produce la intromisión en las actividades primarias de las agroindustrias en nuestra zona, podemos decir que son múltiples. Las modificaciones sociales y espaciales que desencadenan son evidentes, no obstante corresponde precisar que el fenómeno de las alteraciones socioterritoriales no se debe exclusivamente al accionar de las agroindustrias, ya que en este sentido entran en juego un cúmulo de otros factores, pero sí es indudable que estos agentes económicos las incrementan.

En concreto, en nuestro medio las alteraciones mencionadas se manifiestan, en un plano general, en profundas modificaciones de la estructura agraria, de los actores sociales intervinientes y en el deterioro del medio ambiente, entre otras realidades.

Con respecto a los cambios específicos -que las agroindustrias van a tender a acentuar-, podemos puntualizar los siguientes aspectos:

- Una sobrevaluación del precio de la tierra, con la consecuente limitación de la venta de la misma, lo que conlleva un incremento de la disponibilidad de tierra en arrendamiento.

- A pesar de la mayor disponibilidad de tierras para arrendar, la competencia por las mismas también se acrecienta, generando nuevamente- desigualdades en su acceso. De esta manera, las grandes empresas pueden contratar (y pagar el alquiler) por adelantado, consiguiendo así precios más bajos en un contexto en el que los valores de arrendamiento continúan incrementándose notablemente.

- Un incremento del tamaño de las explotaciones y una disminución numérica de las mismas -no así obligadamente de las tierras en propiedad-, como así también de los productores.

Los dos aspectos nombrados van a implicar fuertes alteraciones de la estructura agraria fundiaria.

- Sobreintensificación -acompañada de cuasi monocultivo- en el uso de la tierra y 
expansión de las explotaciones agrícolas en sectores periféricos, con el previsible deterioro del medio ambiente.

- Aparición y consolidación de sectores sociales no tradicionales -que si bien no son agentes productivos estrictamente nuevos, se incrementaron sobremanera en los últimos tiempos-. Éstos van a estar representados por contratistas de servicios, productores capitalistas, contratistas capitalistas y obviamente las mismas agroindustrias.

- Intensificación del proceso de despoblamiento del campo, ya iniciado desde hace muchos años.

- Achicamiento de la PEA Agropecuaria en general, con particular incidencia en los productores residentes en el campo y en los asalariados permanentes y transitorios no especializados.

- Desdibujamiento de las materialidades del paisaje agrario, que se evidencian en casas y puestos abandonados, caminos rurales secundarios en desuso, alambrados caídos, escuelas fuera de servicio, almacenes convertidos en taperas, etc.

Como reflexión final, podemos decir que la tendencia a futuro de la incidencia directa de las agroindustrias en la producción agropecuaria, a nuestro parecer, no son halagüeñas, en el sentido de que cada vez se van a acentuar más las asimetrías ya descriptas, acelerando la concentración del capital y alejando la posibilidad de una distribución social más equitativa de los beneficios que pueda llegar a brindar la tierra.

Asimismo, entendemos que las alternativas que se pueden presentar en la búsqueda más equitativa en provecho de los productores agropecuarios, si bien son difusas y están muy lejos de alcanzarse, quizás pase -como bien puntualizaba un productor entrevistado- por enfrentar al sistema societario agroindustrias-productores "impuesto" por las primeras, mediante la implementación de asociaciones auténticamente horizontales de productores que busquen, todos a la vez, la defensa de su trabajo y producción en base a la potenciación proveniente de la sumatoria de sus capitales y de la unión grupal. Pero por ahora, de acuerdo con las orientaciones económicas predominantes que rigen el quehacer agropecuario del campo argentino, se está seguramente muy lejos de alcanzar esta suerte de utopía.

\section{REFERENCIAS:}

BARSKY, Osvaldo (compilador). El desarrollo agropecuario pampeano. Buenos Aires: Grupo Editor Latinoamericano, 1991.

FORNI, Floreal; TORT, María Isabel. La tecnología y el empleo en un nuevo enfoque del desarrollo agropecuario. El caso argentino. Desarrollo Económico, Buenos Aires, v. 19, n. 76, p. 499-538, enero/marzo 1980.

GUTMAN, Graciela; GATTO, Francisco (compiladores). Agroindustrias en la Argentina. Cambios organizativos y productivos (1970-1990). Buenos Aires: Centro Editor de América Latina, 1990.

HUERGO, Héctor. El modelo agroexportador. Clarín, Buenos Aires, 17 mayo 2003. Clarin Rural, p. 4-5.

PENGUE, Walter. Sustentables, ¿hasta cuándo? Le Monde Diplomatique, Buenos Aires, p. 25-27, mayo 2000 .

PUCCIARELLI, Alfredo. Cambios en la estructura agraria de la pampa bonaerense. Ciclos, Buenos Aires, v. 3, n. 5, año 3, p. 69-90, julio/diciembre 1993.

RODRÍGUEZ, Javier; TEUBAL, Miguel. Ajuste, reestructuración y crisis del agro. Le Monde Diplomatique, Buenos Aires, p. 6-7, diciembre. 2001.

ROFMAN, Alejandro; ROMERO, Luis. Sistema socioeconómico y estructura regional en la Argentina. Buenos Aires: Amorrortu Editores, 1997.

Sociedade \& Natureza, Uberlândia, 20 (2): 121-133, DEZ. 2008 
TEUBAL, Miguel. Globalización y expansión agroindustrial: ¿superación de la pobreza en

América Latina? Buenos Aires: Ediciones Corregidor, 1995.

TEUBAL, Miguel; RODRÍGUEZ Javier. Agro y alimentos en la globalización: una perspectiva crítica. Buenos Aires: Editorial La Colmena, 2003. 